\title{
A case of myocardial infarction due to myocardial bridging alone
}

\author{
Authors: Li Zhou, ${ }^{\mathrm{A}}$ Sheng-yu Li, ${ }^{\mathrm{B}}$ Dong-bao Li ${ }^{\mathrm{C}}$ and Hui Chen ${ }^{\mathrm{C}}$
}

DOI: $10.7861 /$ clinmed.2019-0460

\section{Introduction}

Myocardial bridging (MB) is characterised by a segment of a major epicardial coronary artery tunnelling through the myocardium, which is a frequent congenital anomaly, detected in $15-85 \%$ of autopsy series and in $0.5-33 \%$ of angiographic studies. ${ }^{1,2} \mathrm{MB}$ without critical stenosis is generally considered as a harmless clinical anomaly in coronary angiography (CAG), but in some cases myocardial ischaemia, infarction, vasospasm, cardiac arrhythmias, ventricular rupture and sudden cardiac death have been reported. ${ }^{3-7}$ We describe an acute myocardial infarction in a premenopausal woman due to MB alone, confirmed in a series of angiographic examinations, including optical coherence tomography (OCT).

\section{Case presentation}

A 47-year-old woman was admitted from the outpatients department with complaints of general weakness and low-effort shortness of breath for 3 days after mountain climbing, without typical chest pain. She was premenopausal and had no history of hypertension, diabetes, dyslipidaemia, smoking and had no family history of coronary artery disease. Her body mass index was $23 \mathrm{~kg} / \mathrm{m}^{2}$. No physical abnormalities were detected by clinical examination and blood pressure was 132/86 mmHg.

Her electrocardiography (ECG) on admission showed sinus rhythm with pathologic Q waves, and inverted T waves in precordial leads $V_{1}$ to $V_{4}$ (Fig 1). Creatine kinase-MB was within normal limits $(0.60 \mathrm{ng} / \mathrm{mL}$; normal range $0-6.60 \mathrm{ng} / \mathrm{mL})$, and troponin T was elevated $(0.25 \mathrm{ng} / \mathrm{mL}$; normal range $0-0.03 \mathrm{ng} /$ $\mathrm{mL}$ ). On transthoracic $2 \mathrm{D}$ echocardiography examination, a left ventricular ejection fraction of $66 \%$ and a mild segmental anteroapical hypokinesis were detected.

The posteroanterior chest X-ray showed no pulmonary congestion. Blood tests revealed triglycerides of $2.36 \mathrm{mmol} / \mathrm{L}$, total

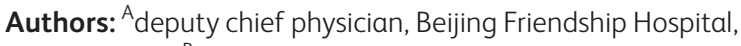
Beijing, China; ${ }^{B}$ resident, Beijing Friendship Hospital, Beijing, China; ${ }^{C}$ medical professor, Beijing Friendship Hospital, Beijing, China cholesterol of $3.72 \mathrm{mmol} / \mathrm{L}$, high-density lipoprotein cholesterol of $0.97 \mathrm{mmol} / \mathrm{L}$, low-density lipoprotein cholesterol of $1.98 \mathrm{mmol} / \mathrm{L}$, fasting blood glucose of $4.01 \mathrm{mmol} / \mathrm{L}$ and glycosylated haemoglobin of $5.2 \%$. Plasma D-dimer level and the activities of protein $\mathrm{S}$ and protein $\mathrm{C}$ were normal. Other chemical and haematological profiles showed no abnormality.

A symptom-limited exercise stress myocardial perfusion tomographic imaging (MPI) was performed 3 days later on a bicycle ergometer with an initial $50 \mathrm{~W}$ load and successive $25 \mathrm{~W}$ increments every 3 minutes until exhaustion. MPI showed a transmural myocardial perfusion defect at anteroapical wall (Fig 2).

On the fifth hospital day, CAG and left ventriculography were performed. The left coronary system was imaged at left and right oblique, right cranial and caudal and anteroposterior cranial positions. CAG revealed obvious MB in the mid segment of the left anterior descending (LAD) coronary artery with compression to $80 \%$ stenosis during systole (Fig 3). A significant 'milking phenomenon' was observed during systole, which was released in diastole. The right coronary artery and circumflex coronary artery were angiographically normal. Left ventriculography showed mild hypokinesis of the apical region. OCT detected

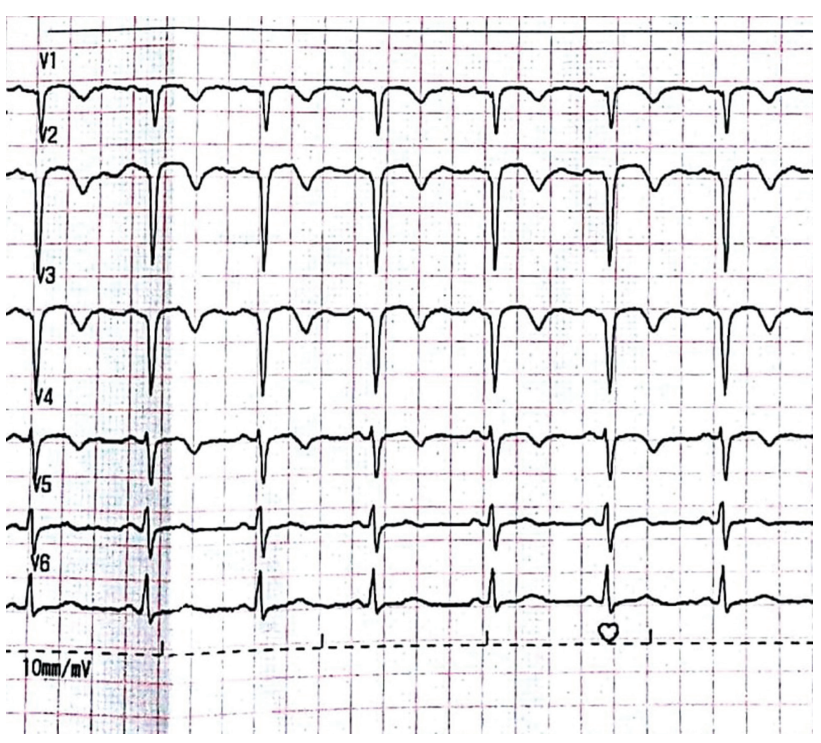

Fig 1. Electrocardiography on admission showing sinus rhythm with pathologic $Q$ waves, and inverted $T$ waves in precordial leads $V_{1}$ to $V_{4}$. 


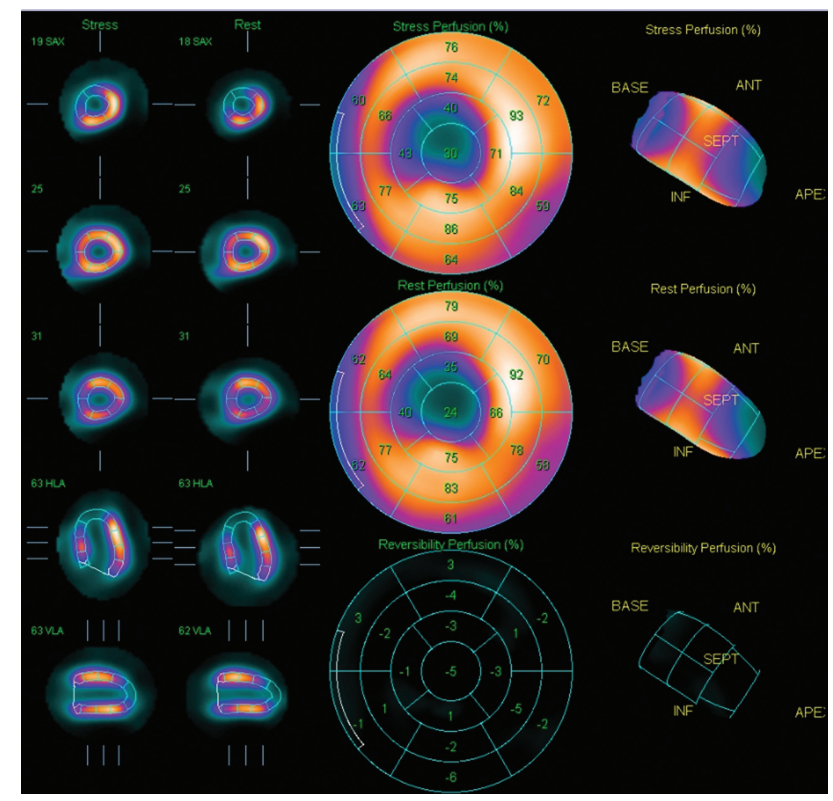

Fig 2. Myocardial perfusion tomographic imaging showing a transmural myocardial perfusion defect at anteroapical wall.

a heterogeneous, generally signal-poor fusiform area with a sharply delineated border closely surrounding the adventitia of the MB segment, without any fixed atherosclerotic stenosis or thrombus (Fig 4a). In addition, OCT revealed an atherosclerotic plaque with a thick fibrous cap in the segment proximal to the $\mathrm{MB}$, but did not reveal any plaque within or distal to the site (Fig 4b). She was treated with perindopril, metoprolol, atorvastatin, aspirin and ticagrelor.

On the seventh hospital day, the patient remained asymptomatic and was discharged. She was instructed on a heart-healthy diet and exercise programme.

When seen 9 months later in the outpatient cardiology clinic, the patient reported compliance with her medications and denied any recurrence of symptoms. A decreased left ventricular systolic function (54\%) and apical hypokinesis were found during followup echocardiography.

\section{Management}

The MB has long been considered benign based on angiographic findings, and patients with an MB in the LAD are given a good long-term prognosis. However, many case studies have indicated a close association between the presence of an MB and coronary heart disease (CHD), malignant arrhythmia, sudden cardiac death and Takotsubo cardiomyopathy. ${ }^{8,9}$ Symptomatic cases of MB have been repeatedly described; 183 cases without significant atherosclerosis have been reported from 1968 to $2008 .^{10}$ Such cases of CHD exhibit angina and myocardial infarction, but most patients have no significant coronary lesion except for the MB around the coronary artery, indicating that the CHD in these cases is induced directly by MB vigorous compression of the coronary artery during cardiac systole, irrespective of the presence of coronary atherosclerosis. ${ }^{10}$ In sports medicine, MB is one of the main causes of sudden cardiac death among young competitive athletes.

A sudden and transient narrowing of the bridged arterial segment caused by vasospasm, tachycardia or forceful muscular contraction might provoke angina and myocardial infarction in a symptomatic patient with an MB. Sometimes an acute thrombus formation within $\mathrm{MB}$ may cause a myocardial infarction. The occurrence of $\mathrm{CHD}$ in patients with an $\mathrm{MB}$ is caused by two distinct mechanisms: direct compression of the LAD by MB contraction and enhancement of the natural history of coronary atherosclerosis in the LAD segment proximal to the MB. ${ }^{11,12}$

Angiography reveals that patients exhibiting more than $75 \%$ narrowing of the LAD from MB compression during cardiac systole experience severe myocardial ischaemia. The magnitude of the compressive force exerted by the MB on the coronary artery is distinctly associated with the anatomical properties of the MB namely its length, thickness and location. ${ }^{13}$ In fact, a longer and/or thicker MB has a significant association with more severe systolic compression. ${ }^{14}$ Furthermore, the location of the MB in patients with angina and myocardial infarction tends to be proximal toward the coronary ostium. The presence of an MB in the LAD
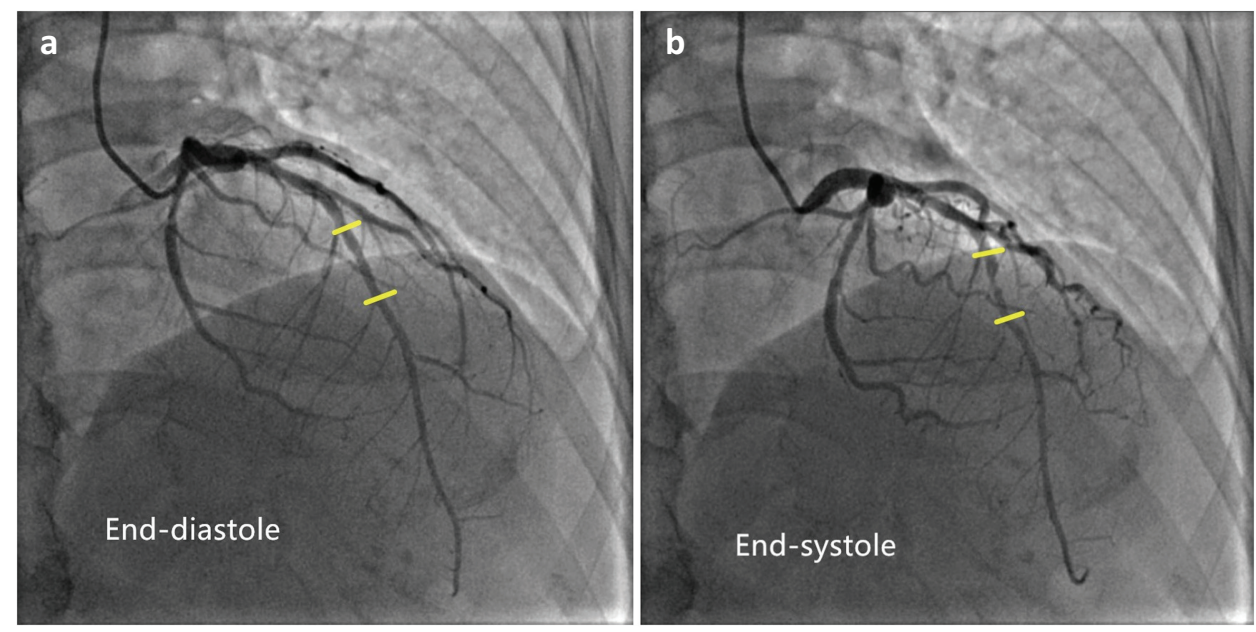

Fig 3. Coronary angiography revealing obvious myocardial bridging in the mid segment of left anterior descending coronary artery with compression to $80 \%$ stenosis during systole. 
Fig 4. Optical coherence tomography. a) Heterogeneous, generally signal-poor fusiform area with a sharply delineated border closely surrounding the adventitia of the MB segment, without any fixed atherosclerotic stenosis or thrombus. b) Atherosclerotic plaque with a thick fibrous cap in the segment proximal to the myocardial bridging.
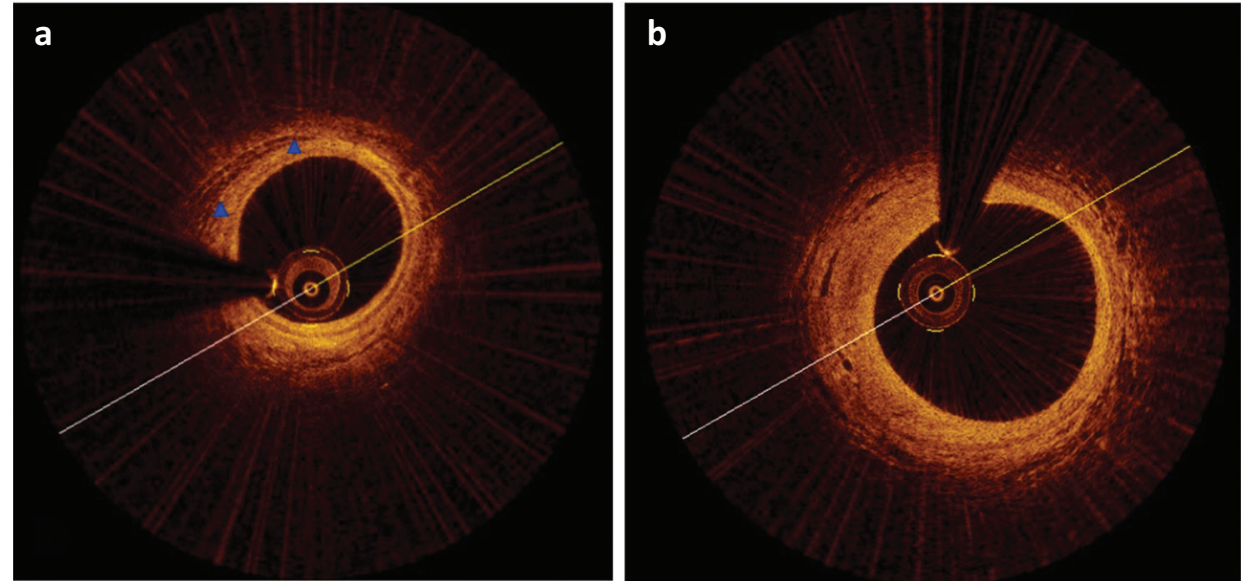

contributes to altered distribution of atherosclerosis within the entire course of the LAD. It is widely accepted that arterial intima beneath $M B$ is significantly spared from atherosclerotic changes, and the LAD segment proximal to the $M B$ is vulnerable to atherosclerosis. ${ }^{11,15}$ Such atherosclerotic evolution in the $\mathrm{LAD}$ intima proximal to the $\mathrm{MB}$ is basically regulated by the anatomical properties of the MB. The more proximal the MB is located to the left coronary ostium, the more turbulent the blood flow becomes in the LAD segment proximal to the MB, thus resulting in the formation of severe atherosclerotic lesions in the segment proximal to the MB. In the present case, OCT detected atherosclerosis but no plaque rupture proximal to the MB segment.

Our case was of a premenopausal woman with MB who developed myocardial infarction without any risk factors of coronary atherosclerotic heart disease. In our patient, we performed angiographic examination and completed the OCT test to confirm the imaging of the LAD lumen with MB but no significant stenosis or thrombus. OCT detected a heterogeneous signal-poor fusiform area with well-delineated borders indicating the existence of the tunnelled artery through the myocardium. According to previous histological evidence, the OCT fusiform area mainly consisted of loose connective tissue in the periarterial space beneath the MB. ${ }^{16}$ Research indicates that the severity of systolic MB compression was attenuated with an increase in the size of the fusiform area, which identified by OCT, was more easily seen during cardiac diastole. ${ }^{17}$ We obtained full imaging pictures of the OCT of the patient, which were seldom reported in previous cases.

Treatment of an MB is restricted to symptomatic patients. It is reported that among the medications used in a long-term clinical follow-up of patients with MB, beta-blockers and calcium channel blockers have been appropriately administered according to the patients' conditions, showing favourable outcomes on most symptoms. ${ }^{11}$ Beta-blocker therapy is the first choice of treatment for symptomatic patients. These drugs reduce the degree of systolic coronary artery narrowing and lengthen the diastolic via their negative inotropic and chronotropic properties. The negative inotropic effect of beta-blockers increases coronary perfusion and alleviates symptoms evoked by the MB. Calcium-channel blockers are an alternative therapeutic agent for patients with a contraindication to beta-blocker therapy, especially in patients with superimposed coronary vasospasm. Antiplatelet drugs and statins can be considered preventive measures in view of the frequent changes in atherosclerosis near the bridge. ${ }^{18}$

The other therapeutic approaches consist of coronary artery angioplasty with stent implantation, coronary artery bypass grafting (CABG) and surgical myotomy of the bridge. Surgical myotomy or $C A B G$ should be limited to patients who have significant symptoms despite drugs. ${ }^{19}$ In most cases, stent placement should not be recommended. Stent implantation in the bridged segment may result in stent thrombosis, restenosis, coronary intimal rupture, stent fracture and aneurysm. ${ }^{20}$

\section{Summary}

We conclude that the main reason for acute myocardial infarction in the present case was the MB alone. Our report indicated that an MB may cause life-threatening events, even in premenopausal woman without any risk factors. Intensive medical treatment is required in patients with a cardiac event associated with MB.

\section{Ethics}

The case report was approved by Beijing Friendship Hospital's committee on human research (approval number 2019-P2-001-01).

\section{Funding}

Funding was provided by the Research Foundation of Beijing Friendship Hospital, Capital Medical University, Beijing (number yyqdkt 2018-13).

\section{References}

1 Alegria JR, Herrmann J, Holmes DR Jr, Lerman A, Rihal CS. Myocardial bridging. Eur Heart J 2005;26:1159-68.

2 Möhlenkamp S, Hort W, Ge J, Erbel R. Update on myocardial bridging. Circulation 2002;106:2616-22.

3 Takamura K, Fujimoto S, Nanjo S et al. Anatomical characteristics of myocardial bridge in patients with myocardial infarction by multi-detector computed tomography. Circ ] 2011;75:642-8.

4 Nishikii-Tachibana M, Pargaonkar VS, Schnittger I et al. Myocardial bridging is associated with exercise-induced ventricular arrhythmia and increases in QT dispersion. Ann Noninvasive Electrocardiol 2018:23:e12492. 
5 Zawiślak B, Dziewierz A, Kmita A et al. Ventricular septal rupture in a patient with non-ST-segment elevation myocardial infarction caused by myocardial bridge. Pol Arch Med Wewn 2015;125:386-8.

6 Feld H, Guadanino V, Hollander G et al. Exercise-induced ventricular tachycardia in association with a myocardial bridge. Chest 1991;99:1295-6.

7 Corrado D, Thiene G, Cocco P, Frescura C. Non-atherosclerotic coronary disease and sudden death in the young. Br Heart ] 1992;68:601-7.

8 Chambers JD Jr, Johns JP, Berndt TB, Davee TS. Myocardial stunning resulting from systolic coronary artery compression by myocardial bridging. Am Heart ] 1994;128:1036-8.

9 Boktor M, Mansi IA, Troxclair S, Modi K. Association of myocardial bridge and Takotsubo cardiomyopathy: A case report and literature review. South Med J 2009;102:957-60.

10 Ishikawa Y, Kawawa Y, Kohda E, Ishii T. Coronary events caused by myocardial bridge. Ann Vasc Dis 2009;2:79-94.

11 Ishikawa Y, Kawawa Y, Kohda E, Shimada K, Ishii T. Significance of the anatomical properties of a myocardial bridge in coronary heart disease. Circ J 2011;75:1559-66.

12 Ishikawa Y, Akasaka Y, Suzuki K et al. Anatomic properties of myocardial bridge predisposing to myocardial infarction. Circulation 2009;120:376-83.

13 Tsujita K, Maehara A, Mintz GS et al. Comparison of angiographic and intravascular ultrasonic detection of myocardial bridging of the left anterior descending coronary artery. Am J Cardiol 2008;102:1608-13.

14 Liu SH, Yang Q, Chen J et al. Myocardial bridging on dual-source computed tomography: Degree of systolic compression of mural coronary artery correlating with length and depth of the myocardial bridge. Clin Imaging 2010;34:83-8

15 Ural E, Bildirici U, Celikyurt $U$ et al. Long-term prognosis of noninterventionally followed patients with isolated myocardial bridge and severe systolic compression of the left anterior descending coronary artery. Clin Cardiol 2009;32:454-7.

16 Ye Z, Lai Y, Mintz GS et al. Fusiform appearance of myocardial bridging detected by OCT. JACC Cardiovasc Imaging 2016;9: 892-4.

17 Nishimiya K, Matsumoto Y, Wang $\mathrm{H}$ et al. Absence of adventitial vasa vasorum formation at the coronary segment with myocardial bridge: An optical coherence tomography study. Int J Cardiol 2018;250:275-7.

18 Kim SS, Jeong MH, Kim HK et al. Long-term clinical course of patients with isolated myocardial bridge. Circ J 2010;74:538-43.

19 Bockeria LA, Sukhanov SG, Orekhova EN et al. Results of coronary artery bypass grafting in myocardial bridging of left anterior descending artery. J Card Surg 2013;28:218-21.

20 Kunamneni PB, Rajdev S, Krishnan P et al. Outcome of intracoronary stenting after failed maximal medical therapy in patients with symptomatic myocardial bridge. Catheter Cardiovasc Interv 2008;71:185-90.

Address for correspondence: Dr Hui Chen, Department of Cardiology, Beijing Friendship Hospital, Capital Medical University, Xi-Cheng district, Yong-An Road, 95 Beijing 100050, China.

Email: 13910710028@163.com

\section{Mental health and wellbeing resource

$$
\text { e }
$$

This new online resource aims to shine a spotlight on the mental health and wellbeing of physicians by opening up the conversation about mental health issues and their impact.

It will help you recognise the warning signs in yourself and others, as well as know about the steps you can take to stay well and seek the right support when you need it.

www.rcplondon.ac.uk/wellbeing 